\title{
A construção de um glossário bilíngue como auxiliar na retenção de vocabulário em um curso de inglês instrumental de curta duração
}

\author{
The construction of a bilingual glossary as an aid in retaining \\ vocabulary in a short course of English for Specific Purpose (ESP)
}

Cândida Salete Rodrigues Melo ${ }^{1}$

\begin{abstract}
Resumo
Este relato apresenta uma experiência de ensino de vocabulário através da construção de um glossário bilíngue, em uma turma de inglês instrumental. $O$ curso foi parte do projeto intitulado FicEmCasa do IFCE, surgido a partir da situação de isolamento social provocada pela chegada do novo coronavírus ao Brasil. Esse fato impediu aulas de forma presencial e impulsionou a criação de novos cursos, dessa vez a distância. Por ser de curta duração (40h), e ter como foco central o conhecimento de vocabulário na língua estudada, a construção de um glossário bilíngue colaborativo foi vista como uma maneira de aumentar a retenção de novas palavras pelos estudantes. 0 recurso é defendido pela abordagem explícita de ensino de vocabulário que serviu de aporte para a atividade. Ao final do curso, os alunos concluíram o glossário proposto.
\end{abstract}

Palavras-chave: Vocabulário. Ensino. Glossário.

\begin{abstract}
This is a report of a vocabulary teaching experience through a bilingual glossary activity with a group of English for Specific Purpose (ESL). The course was part of the project named FicEmCasa, from IFCE, and started from the situation of social isolation caused by the arrival of the new coronavirus in Brazil. This fact prevented face-to-face classes and boosted the creation of new online courses. For being a short course (40h) and having the central focus on vocabulary teaching on the target language, the building of a glossary was chosen as a way to improve the retention of new words by the students. This resource is advocated for the vocabulary teaching explicit approach that was used as basis to the research. At the end of the course, the students finished the glossary building.
\end{abstract}

Keywords: Vocabulary. Teaching. Glossary.

\section{Introdução}

Com a chegada da pandemia da COVID-19 em 2020, no Brasil, o governo do Estado do Ceará suspendeu todas as atividades educacionais presenciais. O IFCE cumpriu essa determinação e suspendeu as aulas em todos os cursos regulares, tanto na modalidade presencial quanto na remota, para evitar que os alunos de baixa renda e lou moradores das áreas rurais fossem prejudicados por não terem acesso à internet para acompanhar as aulas. Desta forma, o IFCE propôs a oferta de cursos

\footnotetext{
${ }^{1}$ Mestre em Linguística Aplicada. Instituto Federal de Educação, Ciência e Tecnologia do Ceará - IFCE (Cândida Salete Rodrigues Melo, Fortaleza, Ceará, Brasil). Orcid:https://orcid.org/0000-0003-3748-4486. E-mail candida.ifce@gmail.com.
} 
remotos na modalidade FIC, dentro do projeto FicEmCasa, disponíveis tanto para alunos da instituição como para membros da comunidade externa. De acordo com a proposta, os professores ofertariam cursos de curta duração, não-regulares, por meio das plataformas Google Classroom ou Moodle. Os estudantes interessados já iriam se inscrever sabendo que o curso seria on-line, ou seja, os alunos necessitariam ter acesso a equipamentos digitais e internet para acompanhar essas aulas, que aconteceriam completamente à distância. Foi a partir daí que decidiu-se ofertar o curso de inglês instrumental apresentado neste relato.

Nos dias atuais, professores, pesquisadores e acadêmicos parecem concordar que o vocabulário é um dos componentes centrais no processo de ensino-aprendizagem de uma Língua Estrangeira (LE). Entende-se, assim, a importância que o conhecimento lexical tem no contexto da leitura em inglês como LE. Considerando esse fato, o relato tem como objetivo apresentar experiência de ensino de vocabulário para uma turma de inglês instrumental que teve como pano de fundo o isolamento social adotado como prevenção de disseminação da pandemia da COVID-19. A ação se deu durante a oferta de um curso de Formação Inicial e Continuada (FIC) do Instituto Federal de Educação, Ciências e Tecnologia do Ceará (IFCE), realizado a distância e utilizando a plataforma digital Google Classroom. Os cursos de categoria FIC, no qual o inglês instrumental se enquadrou, visam capacitar, aperfeiçoar e atualizar profissionais em todos os níveis de escolaridade, nas mais diversas áreas do conhecimento.

0 inglês instrumental foi ofertado como curso de extensão, com 40 horas, no período entre 18/05/2020 a 14/06/2020, de maneira remota, e teve como objetivo apresentar aporte gramatical, de vocabulário e estratégias de leitura, a fim de facilitar a compreensão de textos em inglês. As aulas foram ministradas por uma única professora que ofertou 40 vagas para alunos com ensino médio completo. Dessa oferta, 34 começaram a frequentar, mas apenas 20 concluíram com aprovação. 0 glossário desenvolveu-se como forma de aumentar a aprendizagem de vocabulário. Optou-se, assim, por trabalhar os sentidos de vocabulários novos encontrados pelos alunos nos textos que leram durante todo o período de ensino. A atividade aconteceu paralelamente ao estudo de outros conteúdos previstos no plano de trabalho da disciplina.

A seguir, apresentamos as abordagens teóricas e metodológicas que serviram de alicerce para o desenvolvimento da atividade.

\section{Aporte Teórico}

Para melhor compreender a atividade que será relatada, é necessário termos uma noção básica do ensino de inglês instrumental no Brasil, assim como apresentar como se dá o ensino a distância 
no país e as abordagens de ensino de vocabulário, dentre elas, a que foi utilizada como aporte para nosso relato.

Segundo Vian Jr. (1999), o ensino de inglês instrumental no Brasil começou em 1962 com a publicação do artigo "Some measurable characteristics of modern scientific prose", Barber (1962) (Algumas características mensuráveis de prosa científica moderna). Porém, acredita-se, que o ensino instrumental, de maneira informal, sempre existiu. Basta considerarmos, por exemplo, os contatos entre os impérios grego e romano, nos quais a língua utilizada com os povos conquistados era aprendida com o fim específico de estabelecer relações de dominado/dominante entre as partes, o que já representa um fim instrumental.

Robinson (1980) define ESP (English for Specific Purposes - Inglês para Fins Específicos) como um curso cujo objetivo é o bom desempenho de papéis ocupacionais ou educacionais, baseado numa análise rigorosa das necessidades dos alunos. Kennedy e Bolitho (1984) definem como um tipo de inglês a ser ensinado baseado no interesse e necessidade do aluno. Já Hutchinson e Waters (1987) fazem uma analogia entre o ensino de inglês e uma árvore, sendo o inglês instrumental como um dos ramos de ensino de inglês como língua estrangeira. Para os autores supracitados, o ensino de inglês instrumental é visto como uma abordagem e não como um produto, havendo uma preocupação com as necessidades do aprendiz.

Em Dudley-Evans e St John (1998), uma definição de inglês instrumental deve refletir o fato de que a metodologia instrumental difere da metodologia utilizada no ensino de inglês para fins gerais, uma vez que, numa situação envolvendo o ensino instrumental, o professor passa a ser um consultor, ao passo que o aluno mantém o seu status de especialista em sua área de atuação. Os autores mencionam também, como características absolutas para o inglês instrumental, dentre outras, ser designado para atender às necessidades específicas do aluno e centrado na linguagem (léxico, gramática e registro), habilidades, gêneros textuais e discursos apropriados para essas atividades.

Observa-se assim, a partir das definições apresentadas anteriormente, a existência de preocupações com as necessidades do aluno, assim como a clareza nos objetivos do curso e a existência de conteúdos específicos. Essas características são de grande importância em cursos de inglês instrumental, já que os alunos têm objetivos claramente definidos e querem atingi-los em um curto prazo.

No que se refere ao ensino a distância (EaD), segundo Farias (2019), no Brasil, de uma forma geral, e em uma perspectiva histórica, o EaD apresenta um percurso tímido, alcançando maior celeridade nas últimas décadas e, só nos últimos anos, maior representatividade, principalmente, no tocante à educação superior. 
Sobre as abordagens de ensino de vocabulário que será objeto do relato, pode-se mencionar que, segundo Hulstijn (2011), existem duas abordagens de ensino-aprendizagem de vocabulário: a explícita e a implícita. A aprendizagem explícita refere-se à aprendizagem consciente de fatos ou regularidades do insumo linguístico, aos quais os aprendizes são expostos. Dentre as atividades de aprendizagem explícita, temos exercícios de construção de palavras, tarefas relacionadas com vários tipos de definições e estudo de vocabulário em contexto, mapeamento semântico (mind map) e atividades de preenchimento de informação (information-gap) entre os participantes, cujo foco é o aprendizado de vocabulário (NATION; NEWTON, 1997).

A abordagem implícita ocorre, segundo Zeeland e Schmitt (2013), quando o aprendiz adquire vocabulário sem que tenha sido esse seu objetivo. Essa aprendizagem de vocabulário acontece pelo contexto, ela é um subproduto de outra atividade e não o alvo da cognitiva principal. A aprendizagem de vocabulário através dessa abordagem pode ser incrementada por recursos que façam os aprendizes pesquisarem o significado das palavras conhecidas, processarem sua relação forma-significado e processá-la novamente após a leitura (PETERS; LEUVEN, 2007).

No que se refere ao glossário, Farias (2019) explica que o instrumento é entendido como um recurso que traz definições ou explicações de palavras difíceis que aparecem nas margens dos materiais impressos e em locais pré-determinados da tela dos monitores, em materiais eletrônicos. Conforme a autora, a qualidade e quantidade de informações podem variar conforme os objetivos e público-alvo a serem alcançados.

Apoiado nas informações mencionadas acima sobre o ensino de ESP, com 0 intuito de aumentar a retenção do vocabulário estudado durante o curso, com base na informação de pesquisa de significados e, partindo da abordagem explícita, optou-se por construir um glossário colaborativo. A atividade foi realizada a partir da identificação de palavras desconhecidas dos textos estudados pelos alunos e inferência ou busca de seus sentidos em português. $O$ fato se deu durante o curso de inglês para fins específicos, denominado de inglês instrumental, que será relatado em seguida.

\section{Metodologia}

\subsection{Contexto}

O curso de inglês instrumental no qual a atividade foi realizada foi aberto à comunidade externa em geral e teve como critério de escolaridade a comprovação de ensino médio completo. Das 40 vagas disponibilizadas, foram aceitas 80 inscrições e dessas, foram selecionados os estudantes que fariam 0 curso. Após as matrículas, foram passados os contatos desses alunos para que fossem adicionados à 
sala no Google Classroom, através de seus e-mails. Dos 40 selecionados, 34 aceitaram o convite, mas apenas 25 começaram efetivamente o curso. Por sugestão da própria turma, foi criado um grupo de Whatsapp para comunicação entre eles e a professora que ministraria as aulas. Então, no dia 18 de maio de 2020, deram início as aulas.

\subsection{Desenvolvimento das atividades}

A turma de Inglês Instrumental foi ofertada pelo IFCE em sua página, no link http://web.ficemcasa.ifce.edu.br/. Dividido em quatro semanas, cada atividade do curso deveria corresponder a 10 horas, compreendendo videoaula, atividade de leitura, fórum e atividade avaliativa. De acordo com o plano de trabalho, foram selecionados os seguintes assuntos a serem trabalhados: atividade I, estratégias de leitura; atividade II, tipos de leitura (skimming, scanning, leituras intensiva e extensiva); atividade III, grupos nominais; atividade IV, afixos e tempos verbais (presente, passado e futuro).

Então, com o objetivo de maior apreensão do vocabulário estudado, através da metodologia da aprendizagem explícita em que os alunos, conscientemente, teriam mais uma prática de aprendizagem, decidiu-se por criar um glossário colaborativo que será descrito em seguida.

A atividade se deu a partir da leitura de textos trabalhados durante todo o curso. O material usado foi retirado de livros e sites de jornais disponibilizados na internet, e tratava de assuntos diversos, tais como, Building your resume: resume tips for college students (Construindo seu currículo: dicas de resumo para estudantes de faculdade), How to use a Blender (Como usar um liquidificador) e Sales Representative (Representante de vendas), Don't write off organic food (Não descarte alimentos orgânicos) e, Michelangelo's first painting bought (A primeira obra de Michelangelo comprada). A opção por assuntos distintos se deu pela possível heterogeneidade de formação acadêmica ou técnica dos alunos que poderiam fazer o curso, uma vez que, o critério de seleção seria possuir ensino médio completo, o que possibilita a matrícula de alunos de diversas áreas de atuação.

Durante a leitura dos textos, surgiram muitas palavras desconhecidas pelos alunos, cujos sentidos deveriam ser entendidos pelo contexto em que se encontravam ou com o auxílio do uso de dicionários bilíngues de línguas inglesa e portuguesa. $\mathrm{O}$ aprendiz deveria retirar duas palavras de cada texto e inseri-las no documento colaborativo que os mesmos estavam alimentando para, ao final, formarem um glossário. 
O glossário que trata a atividade relatada foi criado a partir do uso do instrumento Documentos do Google, que pode ser compartilhado e trabalhado em conjunto, de forma on-line. Criou-se em forma de tabela um arquivo que constava de dois campos: um para a palavra em inglês que os alunos retirariam dos textos trabalhados, intitulado English word (palavra em inglês), e outro, para o significado da palavra em português, juntamente ao trecho em que apareceria no texto, intitulado, Portuguese meaning and example (significado em português e exemplo). Tal documento já dispunha de um exemplo que serviria de modelo para os alunos, conforme o quadro abaixo.

\begin{tabular}{|c|l|}
\hline ENGLISH WORD & \multicolumn{1}{|c|}{ PORTUGUESE MEANING AND EXAMPLE } \\
\hline AWARDS & $\begin{array}{l}\text { PREMIAÇÕES. Ex.: include academic awards (incluir premiações } \\
\text { acadêmicas) }\end{array}$ \\
\hline
\end{tabular}

\section{Quadro 1. Modelo do glossário.}

Fonte: autora

A construção do glossário não teria caráter avaliativo, apenas um exercício extra que seria compartilhado com o grupo ao final do curso. Como as palavras seriam depositadas conforme retiradas dos textos, ficariam em ordem aleatória, e ao final seriam disponibilizadas em ordem alfabética pelo professor proponente.

O glossário (Anexo I deste relato) foi finalizado com 87 palavras, retiradas de 5 textos trabalhados. Como o curso foi concluído com 20 alunos, caso todos tivessem retirado as duas palavras sugeridas, teríamos ao final 200 palavras. Atribui-se essa quantidade abaixo do esperado pelo fato da atividade não ser avaliativa. Após a conclusão, o trabalho foi revisado e colocado em ordem alfabética formatado e compartilhado com a turma.

\section{Considerações Finais}

Conforme demonstram pesquisas prévias, a aprendizagem de vocabulário através da abordagem explícita pode ser incrementada através de tarefas de estudo de vocabulário em contexto. De posse desse conhecimento, foi decidido criar, com os alunos do curso de inglês instrumental a distância, um glossário colaborativo de palavras desconhecidas por eles, retiradas dos textos os quais os estudantes trabalharam durante as aulas. Ao final do curso, o instrumento foi concluído pelo grupo. Embora não tenha sido feita uma coleta de dados posterior à realização da atividade, com o objetivo de constatar os resultados de aumento de aprendizagem, admite-se que essa tarefa coletiva tenha 
adicionado mais contato dos estudantes com uma parte importante no aprendizado de uma língua estrangeira que é o conhecimento de vocabulários na língua-alvo, melhorando, assim, a retenção dos conhecimentos adquiridos no curso.

\section{Referências}

DUDLEY-EVANS T; ST. JOHN, M. Developments in English for specific purposes. Cambridge University Press, 1998.

FARIAS, L. G. B. Educação profissional técnica de nível médio a distância no Brasil: meta-análise da regulamentação dos estados do Nordeste, Mestrado em Pedagogia do e-Learning, Universidade Aberta, 2019.

HULSTIJN, J. H. Incidental learning in second language acquisition. In: C. A. CHAPELLE. (Ed.). The encyclopedia of applied linguistics. Wiley-Blackwell, 2011.

HUTCHINSON, T.; WATERS, A. English for specific purposes. Cambridge, 1987.

KENNEDY, C.; BOLITHO, R. English for specific purposes, Macmillan, 1984

NATION, I. S. P.; NEWTON, J. Teaching vocabulary. In: COADY, J.; HUCKIN, T. Second Language Vocabulary Acquisition. Cambridge University Press, 1997.

PETERS, E.; LEUVEN, K. Manipulating L2 learners online dictionary use and its effect in L2 word retention. Language Learning \& Technology, v. 11, n. 2, 2007.

PROCÓPIO, R. B. O uso do glossário hipermídia no ensino-aprendizagem de vocabulário nos níveis elementar e intermediário de proficiência em inglês, Programa de Pós- graduação em Linguística-UFJF, 2016.

ROBINSON, J. P. The changing reading habits of the American public. Journal of communication, v. 30 , n.1, 1980.

VIAN JR. O. Inglês Instrumental, Inglês para negócios e inglês instrumental para negócios. DELTA, vol. 15. São Paulo, 1999.

ZEELAND, H.; SCHMITT, N. Incidental vocabulary acquisition through L2 listening: a dimension's approach. System, v. 41, n. 3, 2013. 


\section{ANEXO I}

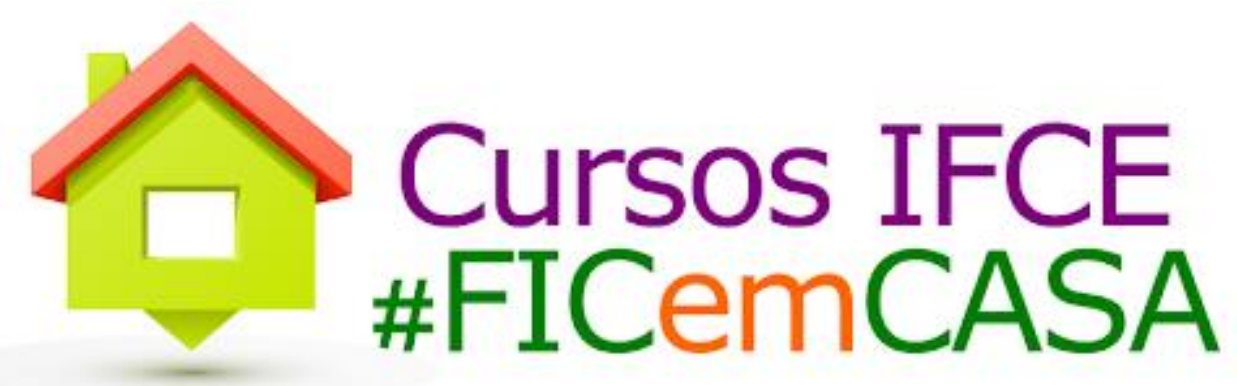

GLOSSÁRIO DE INGLÊS INSTRUMENTAL

\begin{tabular}{|l|l|}
\hline ACCOLADES & ELOGIOS. EX.: as well as special accolades... (bem como elogios especiais). \\
\hline ALMOST & $\begin{array}{l}\text { QUASE. Ex.: You will almost certainly get caught in any lies... (você quase } \\
\text { certamente será pego em qualquer mentira...). }\end{array}$ \\
\hline ALSO & TAMBÉM. Ex.: but also that it was... (mas também que era...) \\
\hline ANOTHER & $\begin{array}{l}\text { OUTRA. Ex.: This is another 'filler' section that isn't required (esta é outra seção } \\
\text { de preenchimento que não é necessária). }\end{array}$ \\
\hline APPLICATION & $\begin{array}{l}\text { INSCRIÇÃO. Ex.: during the application phase, some will ask that these contacts } \\
\text { be included with your resume (durante a fase de inscrição, alguns irão solicitar } \\
\text { que esses contatos sejam incluídos no seu currículo) }\end{array}$ \\
\hline ATTENDING & $\begin{array}{l}\text { PARTICIPAR. Ex.: Attending conferences to demonstrate products and find new } \\
\text { customers. (Participar de conferências para demonstrar produtos e encontrar } \\
\text { novOs clientes) }\end{array}$ \\
\hline AVERAGE & $\begin{array}{l}\text { MÉDIA. Ex.: "Did you know that the average industrially-produced..." (Você sabia } \\
\text { que a média produzida industrialmente...) }\end{array}$ \\
\hline AVOID & $\begin{array}{l}\text { EVITAR. Ex.: "...to avoid the risk of electrical shock." (...para evitar o risco de } \\
\text { choque elétrico). }\end{array}$ \\
\hline AWARDS & PREMIAÇÕES. Ex.: include academic awards (incluir premiações acadêmicas) \\
\hline BANNED & $\begin{array}{l}\text { PROIBIDO. EX.: "Artificial nitrogen fertilizer is banned in organic farming". (O } \\
\text { fertilizante artificial de nitrogênio é proibido na agricultura orgânica) }\end{array}$ \\
\hline BENEFITS & $\begin{array}{l}\text { BENEFíCIOS. Ex.: "of the range of benefits that organics farming and food } \\
\text { deliver". (a gama de benefícios que a agricultura e os alimentos orgânicos } \\
\text { proporcionam). }\end{array}$ \\
\hline
\end{tabular}

LínguaTec, Instituto Federal de Educação, Ciência e Tecnologia do Rio Grande do Sul, Bento Gonçalves 


\begin{tabular}{|c|c|}
\hline BETWEEN & $\begin{array}{l}\text { ENTRE. Ex.: ...little difference between the two (... pouca diferença entre os } \\
\text { dois). }\end{array}$ \\
\hline BEYOND & $\begin{array}{l}\text { ALÉM. Ex.: "which stretch well beyond the nutritional". (que se estendem muito } \\
\text { além o nutricional). }\end{array}$ \\
\hline BIRDS & $\begin{array}{l}\text { PÁSSAROS. Ex.: "organic farms have on average } 30 \text { per cent more species and } \\
50 \text { per cent more overall numbers of wildlife such as birds" (fazendas orgânicas } \\
\text { têm em média } 30 \% \text { mais espécies e } 50 \% \text { mais números gerais de vida } \\
\text { selvagem, como pássaros) }\end{array}$ \\
\hline BLENDER & LIQUIDIFICADOR. Ex.: How to use a Blender (como usar um liquidificador). \\
\hline BULK & $\begin{array}{l}\text { A GRANEL. Ex.: The latter representatives typically sell office supplies, bulk } \\
\text { food... (estes últimos representantes normalmente vendem material de } \\
\text { escritório, alimentos a granel...) }\end{array}$ \\
\hline CAREFULLY & $\begin{array}{l}\text { CUIDADOSAMENTE. Ex.: "departamento de conservação de pinturas o limpou } \\
\text { cuidadosamente" }\end{array}$ \\
\hline CAUGHT & $\begin{array}{l}\text { APANHADO. Ex.: You Will almost certainly get caught. (Você quase certamente } \\
\text { será pego) }\end{array}$ \\
\hline CEILING & $\begin{array}{l}\text { TETO. Ex.: "... the famous scenes on the ceiling and wall of Rome's Sistine } \\
\text { Chopel. (as famosas cenas no teto e na parede da Capela Sistina De Roma) }\end{array}$ \\
\hline CENTURY & $\begin{array}{l}\text { SÉCULO. Ex.: A rare treasure that was tucked away and doubted as authentic } \\
\text { for more than a century. (um raro tesouro que descolado e duvidoso autêntico } \\
\text { por mais de um século.) }\end{array}$ \\
\hline CHAIRMAN & PRESIDENTE Ex.: "department chairman" (presidente do departamento) \\
\hline CHEMICALS & $\begin{array}{l}\text { PRODUTOS QUIMICOS. Ex.: It completely failed to address the long-term } \\
\text { effects of farming chemicals on human health. (Falhou completamente em } \\
\text { abordar os efeitos a longo prazo dos produtos químicos agrícolas na saúde } \\
\text { humana.) }\end{array}$ \\
\hline COLLEGE & $\begin{array}{l}\text { FACULDADE. Ex.: depending on how many years of college you've completed } \\
\text { (dependendo de quantos anos de faculdade você concluiu) }\end{array}$ \\
\hline COMPASSION & $\begin{array}{l}\text { COMPAIXÃO. Ex.: Compassion in World Farming (Compaixão na agricultura } \\
\text { mundial) }\end{array}$ \\
\hline COVERED & $\begin{array}{l}\text { COBERTO, REVESTIDO. Ex.: Sweet, crunchy, nutritious...and covered in } \\
\text { chemicals. (Doce, crocante, nutritivo ... e coberto de produtos químicos.) }\end{array}$ \\
\hline COWORKERS & $\begin{array}{l}\text { COLEGAS DE TRABALHO. Ex.: coworkers or Other reliable sources... (colegas } \\
\text { de trabalho e outras fontes confiáveis) }\end{array}$ \\
\hline DAMP & ÚMIDO. Ex.: Wipe it down with a damp. (Limpe-o com um pano úmido) \\
\hline
\end{tabular}




\begin{tabular}{|c|c|}
\hline DANGEROUS & $\begin{array}{l}\text { PERIGOSO. Ex.: There is also less dangerous waste on organic farms than on } \\
\text { non-organic farms. (Também há menos resíduos perigosos em fazendas } \\
\text { orgânicas do que em fazendas não orgânicas. ) }\end{array}$ \\
\hline DEALER & $\begin{array}{l}\text { NEGOCIANTE. Ex.: Last summer an art dealer bought it for nearly } \$ 2 \text { million at } \\
\text { a Sotheby's auction and then took it to New York's Metropolitan Museum of Art. } \\
\text { (No verão passado, um negociante de arte comprou por quase US } \$ 2 \text { milhões } \\
\text { em um leilão de Sotheby's e depois levou pro Metropolitan Museum of Art de } \\
\text { Nova York). }\end{array}$ \\
\hline DEGREES & $\begin{array}{l}\text { DIPLOMAS, GRADUAÇÕES. Ex.: List any college degrees that you've earned } \\
\text { here (Liste todos os diplomas que você possui aqui) }\end{array}$ \\
\hline DISCLOSE & $\begin{array}{l}\text { DIVULGAR. Ex.: The museum declined to disclose how much it paid for "The } \\
\text { Torment of Saint Anthony,". (O museu se recusou a divulgar quanto pagou por } \\
\text { "O tormento de Santo Antônio".) }\end{array}$ \\
\hline EARLIEST & $\begin{array}{l}\text { PRIMEIRO. Ex.: a Michelangelo painting after acquiring his earliest known } \\
\text { work... (uma pintura após adquirir seu primeiro trabalho conhecido...) }\end{array}$ \\
\hline ENVIRONMENT & $\begin{array}{l}\text { MEIO AMBIENTE. Ex.: The environmental advantages are self-evident... (as } \\
\text { vantagens ao meio ambiente são evidentes...). }\end{array}$ \\
\hline ENVIRONMENTAL & $\begin{array}{l}\text { AMBIENTAIS. Ex.: The environmental advantages are self-evident (As } \\
\text { vantagens ambientais são evidentes) }\end{array}$ \\
\hline FARMING & $\begin{array}{l}\text { AGRICULTURA. Ex.: benefits that organic farming... (benefícios que a } \\
\text { agricultura orgânica...) }\end{array}$ \\
\hline FARMS & FAZENDA. Ex.: “...on non-organic farming.” (...em fazendas não-orgânica). \\
\hline FEAR & TEMER. Ex.: Many students fear that... (Muitos estudantes temem que...) \\
\hline FILL & $\begin{array}{l}\text { PREENCHER. Ex.: “... their resumés to fill blank space”. (...seus currículos para } \\
\text { preencher os espaços) }\end{array}$ \\
\hline FOREIGN & $\begin{array}{l}\text { ESTRANGEIRA. Ex.: foreign language skills or creative skills (habilidades } \\
\text { linguísticas estrangeiras ou habilidades criativas) }\end{array}$ \\
\hline FORGOTTEN & $\begin{array}{l}\text { ESQUECIDO. Ex.: "... the painting was kept privately and largely forgotten in the } \\
\text { art world ..." (... a pintura foi mantida em particular e amplamente esquecida no } \\
\text { mundo da arte, ...) }\end{array}$ \\
\hline GENERATION & $\begin{array}{l}\text { GERAÇÃO Ex.: "The generations of dirt and paint build-up had obscured the } \\
\text { painting's identity" (As gerações de sujeira e acúmulo de tinta obscureceram a } \\
\text { identidade da pintura) }\end{array}$ \\
\hline GOALS & METAS. Ex.: describe your goals (descreva suas metas ). \\
\hline
\end{tabular}




\begin{tabular}{|c|c|}
\hline GREATEST & $\begin{array}{l}\text { MAIOR. Ex.: This is one of the greatest rediscoveries in the history of art,". ("Esta } \\
\text { é uma das maiores redescobertas da história da arte".) }\end{array}$ \\
\hline HEADING & $\begin{array}{l}\text { TÍTULO/CABEÇALHO. Ex.: There should always be a heading at the top of your } \\
\text { resumé that includes your name, mailing address, phone number and email } \\
\text { address. (Sempre deve haver um cabeçalho no topo do seu currículo que inclua } \\
\text { seu nome, endereço para correspondência, número de telefone e endereço de } \\
\text { e-mail). }\end{array}$ \\
\hline HEALTH & $\begin{array}{l}\text { SAÚDE. Ex.: For our own health and the health planet, organic food and farming } \\
\text { will play a big part in a sustainable food and farming future. (Para nossa própria } \\
\text { saúde e a saúde do planeta, alimentos orgânicos e a agricultura terão um grande } \\
\text { papel em um futuro sustentável nos alimentos e na agricultura.) }\end{array}$ \\
\hline HIGHLIGHTING & $\begin{array}{l}\text { DESTACANDO. Ex.: highlighting only relevant duties. (destacando apenas os } \\
\text { deveres relevantes) }\end{array}$ \\
\hline HUNCH & $\begin{array}{l}\text { PALPITE, INTUIÇÃO. Ex.: "where one department chairman shared his hunch } \\
\text { that it was the work of the Renaissance artist" (onde um chefe de departamento } \\
\text { compartilhou seu palpite de que era obra do artista renascentista) }\end{array}$ \\
\hline INTERNSHIPS & $\begin{array}{l}\text { ESTÁGIOS. Etapa de aquisição de experiência profissional ou acadêmica. Ex.: } \\
\text { Keep job descriptions as short as possible, highlighting only relevant duties. } \\
\text { (Mantenha as descrições de cargo o mais curtas possível, destacando apenas } \\
\text { deveres relevantes) }\end{array}$ \\
\hline LAYERS & $\begin{array}{l}\text { CAMADAS. Ex.: "As well as paints layers..." (Bem como as camadas de } \\
\text { tintas..."). }\end{array}$ \\
\hline LEARNING & $\begin{array}{l}\text { APRENDENDO. Ex.: "Learning a potential customer's specific business } \\
\text { regulations". (Aprendendo os regulamentos comerciais específicos de um } \\
\text { cliente em potencial) }\end{array}$ \\
\hline LESS & MENOS. Ex.: There is also less. (Também há menos) \\
\hline LOOSEN & $\begin{array}{l}\text { AFROUXAR. Ex.: And liquid gradually if needed to loosen. (E líquido } \\
\text { gradualmente, se necessário, para afrouxar) }\end{array}$ \\
\hline LOWEST & $\begin{array}{l}\text { MAIS BAIXO. Ex.: Begin processing on the lowest speed setting, then ramp up } \\
\text { as needed. (Comece o processamento na configuração de velocidade mais } \\
\text { baixa e, em seguida, aumente conforme necessário.) }\end{array}$ \\
\hline LOYALTY & $\begin{array}{l}\text { LEALDADE. Ex.: Getting customers involved in incentive programs to increase } \\
\text { loyalty and retention. (envolvendo os clientes em programas de incentivo para } \\
\text { aumentar a lealdade e retenção. }\end{array}$ \\
\hline NEEDED & $\begin{array}{l}\text { NECESSÁRIO. Ex.: Add liquid gradually if needed to loosen a thick mixture and } \\
\text { create a smooth product. (Adicione líquido gradualmente, se necessário, para } \\
\text { soltar uma mistura espessa e criar um produto suave) }\end{array}$ \\
\hline
\end{tabular}




\begin{tabular}{|c|c|}
\hline $\begin{array}{l}\text { OFFICE } \\
\text { SUPPLIES }\end{array}$ & $\begin{array}{l}\text { MATERIAL DE ESCRITÓRIO. Ex.: The latter representatives typically sell office } \\
\text { supplies, bulk food, and even medial equipment right from wholesalers. (Estes } \\
\text { últimos representantes normalmente vendem material de escritório, alimentos a } \\
\text { granel e até equipamentos mediais diretamente do atacadista). }\end{array}$ \\
\hline OPPORTUNITY & $\begin{array}{l}\text { OPORTUNIDADE. Ex.: "That's why this is such an extraordinary opportunity. (É } \\
\text { por isso que essa é uma oportunidade tão extraordinária). }\end{array}$ \\
\hline OUTFIT & $\begin{array}{l}\text { ROUPAS, mas no contesto EQUIPAMENTOS. Ex.: All outfits come with } \\
\text { detachable parts. (Todos os equipamentos vêm com peças destacáveis) }\end{array}$ \\
\hline OVERALL & $\begin{array}{l}\text { NO GERAL. Ex.: More overall numbers of wildlife such as birds. (Maior número } \\
\text { geral de animais selvagens, como pássaros) }\end{array}$ \\
\hline PAID & $\begin{array}{l}\text { PAGOU. Ex.: The museum declined to disclose how much it paid. (O museu se } \\
\text { recusou a divulgar quanto pagou) }\end{array}$ \\
\hline PURCHASING & $\begin{array}{l}\text { COMPRAS. Ex.: "...others use business directories to contact purchasing } \\
\text { agents..." (outros usam diretórios de negócios para contatar agentes de } \\
\text { compras) }\end{array}$ \\
\hline PUSH & $\begin{array}{l}\text { EMPURRAR. Ex.: To stir or push down the ingredients. } \\
\text { (Para agitar ou empurrar os ingredientes para baixo.) }\end{array}$ \\
\hline RECOGNIZED & $\begin{array}{l}\text { RECONHECEU. Ex.: he recognized experts on animal welfare, says organic } \\
\text { farming has the potential for the highest animal welfare standards. } \\
\text { (ele reconheceu especialistas em bem-estar animal, afirma a agricultura } \\
\text { orgânica que tem o potencial para os mais altos padrões de bem-estar animal) }\end{array}$ \\
\hline REDISCOVERIES & $\begin{array}{l}\text { REDESCOBERTAS. Ex.: "This is one of the greatest rediscoveries in the history } \\
\text { of art". (Esta é uma das maiores redescobertas da história da arte) }\end{array}$ \\
\hline RESEARCHERS & $\begin{array}{l}\text { PESQUISADORES. Ex.: But with closer reading the researchers... (mas com } \\
\text { uma leitura mais atenta, os pesquisadores...) }\end{array}$ \\
\hline RESUMÉ & CURRÍCULO. Ex.: resume content (conteúdo do resumo) \\
\hline SAFE & SEGURO. Ex.: $100 \%$ safe for work first. ( $100 \%$ seguro para trabalhar primeiro) \\
\hline SALES & $\begin{array}{l}\text { VENDAS/ VENDEDORES (SALES REPRESENTATIVES). Ex.: "Many sales } \\
\text { representatives sell home products to individuals..." (muitos vendedores (sales } \\
\text { representatives) vendem produtos de casa para pessoas...) }\end{array}$ \\
\hline SCALY & $\begin{array}{l}\text { ESCAMOSO. EX.: "...that depicts scaly, horned, winged demons trying to pull the } \\
\text { Saint out of the sky". ("...que retrata demônios escamosos com chifres alado } \\
\text { tentando puxar o Santo para fora do céu"). }\end{array}$ \\
\hline SELL & $\begin{array}{l}\text { VENDER. Ex.: "The latter representatives typically sell office supplies". (os } \\
\text { últimos representantes vendem material de escritorio...) }\end{array}$ \\
\hline
\end{tabular}




\begin{tabular}{|c|c|}
\hline SHOULD & $\begin{array}{l}\text { DEVE. Ex.: should be cut into small pieces. (deve ser cortado em pequenos } \\
\text { pedaços). }\end{array}$ \\
\hline SKILLS & $\begin{array}{l}\text { HABILIDADES. Ex.: This is your opportunity to draw attention to any important } \\
\text { professional skills that aren't explicit in your education or experience information. } \\
\text { (Esta é a sua oportunidade de chamar a atenção para quaisquer habilidades } \\
\text { profissionais importantes que não sejam explícitas em sua educação ou } \\
\text { informações da experiência). }\end{array}$ \\
\hline SPRAYED & $\begin{array}{l}\text { PULVERISADO. Ex.: "Did you know that the average industrially-produced apple } \\
\text { may have been sprayed up } 16 \text { times with } 30 \text { different chemicals?" (Você sabia } \\
\text { que a maçã produzida industrialmente, em média, pode ter sido pulverizada até } \\
16 \text { vezes com produtos químicos?) }\end{array}$ \\
\hline STANDARDS & $\begin{array}{l}\text { PADRÕES. Ex.: "...the food standards Agency's report..." (...o relatório da } \\
\text { agência de padrões alimentares...). }\end{array}$ \\
\hline SUBMIT & $\begin{array}{l}\text { ENVIAR, SUBMETER. Ex.: Submit cover letters. (enviar cartas de } \\
\text { apresentação). }\end{array}$ \\
\hline SUCH & $\begin{array}{l}\text { TAL COMO. Ex.: "such as professional certifications" (tal como certificações } \\
\text { profissionais) }\end{array}$ \\
\hline SURFACED & $\begin{array}{l}\text { SURGIRAM, VIERAM À TONA. Ex.: But some questions about is authenticity } \\
\text { has surfaced though the years. (Mas algumas perguntas sobre a autenticidade } \\
\text { surgiram nos últimos anos) }\end{array}$ \\
\hline THICK & $\begin{array}{l}\text { ESPESSA. Ex.: "...if needed to loosen a thick mixture..." (...se necessário para } \\
\text { afrouxar uma mistura espessa...). }\end{array}$ \\
\hline TO FILL & $\begin{array}{l}\text { PREENCHER. Ex.: but it can be useful to fill up space if necessary (mas pode } \\
\text { ser útil preencher espaço, se necessário) }\end{array}$ \\
\hline TRAVELLING & $\begin{array}{l}\text { ITINERANTE / VIAJANDO. Ex.: Lee said he may loan the painting to other } \\
\text { museums later for travelling exhibits. (Lee disse que pode emprestar a pintura a } \\
\text { outros museus posteriormente para exposições itinerantes.) }\end{array}$ \\
\hline TRYING & TENTANDO. Ex.: demons trying to pull... (demônios tentando puxar...) \\
\hline UNFINISHED & $\begin{array}{l}\text { INACABADOS. Ex.: "... and two of them are unfinished". (e dois deles estão } \\
\text { inacabados). }\end{array}$ \\
\hline WARM & $\begin{array}{l}\text { QUENTE. Ex.: Take breaks if cup becomes very warm when touched. (faça } \\
\text { pausas se o corpo ficar muito quente ao ser tocado) }\end{array}$ \\
\hline WASTE & $\begin{array}{l}\text { DESPERDÍCIO, RESÍDUOS. Ex.: "There is also less dangerous waste on } \\
\text { organic farms than on non-organic farms." (Também há menos resíduos } \\
\text { perigosos em fazendas orgânicas do que em fazendas não orgânicas). }\end{array}$ \\
\hline
\end{tabular}




\begin{tabular}{|l|l|}
\hline WHOLESALERS & $\begin{array}{l}\text { ATACADISTA. Ex.: medial equipment right from whole salers (equipamento } \\
\text { medial direto dos atacadistas...) }\end{array}$ \\
\hline WILDLIFE & $\begin{array}{l}\text { VIDA SELVAGEM. Ex.: ...can have severe impacts on wildlife. (...pode ter } \\
\text { impactos graves sobre a vida selvagem). }\end{array}$ \\
\hline WISER & $\begin{array}{l}\text { MAIS SÁBIO. Ex.: Agency's report on organic farming would leave you none the } \\
\text { wiser. (o relatório da agência sobre agricultura orgânica não deixa você mais } \\
\text { sábio) }\end{array}$ \\
\hline
\end{tabular}

Data de submissão: 10/11/2020. Data de aprovação: 13/04/2021. 